01

\title{
Спектры собственных волн анизотропного цилиндрического твердотельного волновода
}

\author{
(C) Ю.О. Аверков, ${ }^{1,2}$ Ю.В. Прокопенко, ${ }^{1,3}$ В. М. Яковенко ${ }^{1}$ \\ ${ }^{1}$ Институт радиофизики и электроники им. А.Я. Усикова НАН Украины, \\ 61085 Харьков, Украина \\ ${ }^{2}$ Харьковский национальный университет им. В.Н. Каразина, \\ 61022 Харьков, Украина \\ ${ }^{3}$ Харьковский национальный университет радиоэлектроники, \\ 61166 Харьков, Украина \\ e-mail: yuriyaverkov@gmail.com; prokopen@ire.kharkov.ua
}

(Поступило в Редакцию 19 июля 2017 г.)

Теоретически исследованы дисперсионные свойства собственных волн анизотропного цилиндрического твердотельного волновода, компоненты тензора диэлектрической проницаемости которого обладают частотной дисперсией. Приведена классификация волн. Обнаружены собственные объемно-поверхностные волны $E$-типа, обладающие отрицательными групповыми скоростями. Определены условия их существования. Показано существование в волноводе собственных поверхностных волн $E$-типа и псевдоповерхностных волн $E$ - и $H$-типов.

DOI: 10.21883/JTF.2019.01.46954.2438

\section{Введение}

В работе [1] было обнаружено излучение электромагнитных волн в миллиметровом диапазоне системой, в которой многоструйный цилиндрический поток электронов пролетает над изотропным диэлектрическим цилиндром. Неустойчивость трубчатого электронного пучка при взаимодействии с плазмоподобной средой исследована в работе [2]. В работе [3] развита теория неустойчивости нерелятивистского бесконечно тонкого трубчатого электронного пучка, движущегося в вакууме над диэлектрическим цилиндром. Для понимания природы возникновения неустойчивости при взаимодействии потока заряженных частиц с цилиндрическим твердотельным волноводом особое внимание заслуживают исследования возможности существования в нем собственных волн с отрицательными групповыми скоростями. Большой интерес представляют пучковые неустойчивости, которые возникают в электродинамических системах, содержащих анизотропные диспергирующие среды.

В настоящей работе теоретически исследуются свойства собственных волн анизотропного цилиндрического твердотельного волновода, компоненты тензора диэлектрической проницаемости которого обладают частотной дисперсией. Целью является определение условий существования собственных волн, обладающих аномальной дисперсией.

\section{Постановка задачи и основные уравнения}

Рассмотрим немагнитный цилиндрический твердотельный волновод, который изготовлен из анизотропного одноосного монокристалла так, что его ось анизо- тропии ориентирована параллельно продольной оси симметрии $Z$ цилиндра. Волновод с радиусом $\rho_{0}$ расположен в вакууме. Электромагнитные свойства сред внутри и вне цилиндра описываются уравнениями Максвелла

$$
\operatorname{rot} \mathbf{H}(\mathbf{r}, t)=\frac{1}{c} \frac{\partial}{\partial t} \mathbf{D}(\mathbf{r}, t), \operatorname{rot} \mathbf{E}(\mathbf{r}, t)=-\frac{1}{c} \frac{\partial}{\partial t} \mathbf{H}(\mathbf{r}, t),
$$

где $\mathbf{H}(\mathbf{r}, t)$ и $\mathbf{E}(\mathbf{r}, t)$ - напряженности магнитного и электрического полей соответственно. Вектор электрической индукции $\mathbf{D}(\mathbf{r}, t)$ связан с вектором $\mathbf{E}(\mathbf{r}, t)$ материальным уравнением $[4,5]$

$$
D_{i}(\mathbf{r}, t)=\int_{-\infty}^{t} \hat{\varepsilon}_{i j}\left(t-t^{\prime}\right) E_{j}\left(\mathbf{r}, t^{\prime}\right) d t^{\prime},
$$

где $\hat{\varepsilon}_{i j}\left(t-t^{\prime}\right)-$ функция влияния, характеризующая эффективность передачи действия поля из момента времени $t^{\prime}$ в момент $t$ [5], индексы $i$ и $j$ соответствуют одному из направлений вдоль осей координат $\rho, \varphi$ и $z$, причем по индексу $j$ производится суммирование с перебором всех направлений. В вакууме имеем $\varepsilon_{i j}\left(t-t^{\prime}\right)=\delta_{i j} \delta\left(t-t^{\prime}\right)$, где $\delta_{i j}-$ символ Кронекера и $\delta\left(t-t^{\prime}\right)$ - дельта-функция Дирака.

Векторы напряженностей электромагнитного поля собственных волн цилиндрического твердотельного волновода представим в виде

$$
\begin{aligned}
\mathbf{E}(\mathbf{r}, t) & =\sum_{n=-\infty}^{\infty} \int_{-\infty}^{\infty} \int_{-\infty}^{\infty} \mathbf{E}_{n}\left(\rho, q_{z}, \omega\right) \\
& \times \exp \left[i\left(q_{z} z+n \varphi-\omega t\right) d q_{z} d \omega\right. \\
\mathbf{H}(\mathbf{r}, t) & =\sum_{n=-\infty}^{\infty} \int_{-\infty}^{\infty} \int_{-\infty}^{\infty} \mathbf{H}_{n}\left(\rho, q_{z}, \omega\right) \\
& \times \exp \left[i\left(q_{z} z+n \varphi-\omega t\right) d q_{z} d \omega,\right.
\end{aligned}
$$


где $q_{z}$ - продольное (аксиальное) волновое число, $\omega$ - частота парциальной волны, $|n|$ - азимутальный модовый индекс. Тогда имеем

$$
D_{i}\left(\rho, q_{z}, \omega\right)=\varepsilon_{i j}(\omega) E_{j}\left(\rho, q_{z}, \omega\right),
$$

где $\varepsilon_{i j}(\omega)=\int_{0}^{\infty} \varepsilon_{i j}(\tau) \exp (i \omega \tau) d \tau$ - компоненты тензора диэлектрической проницаемости среды при переборе индексов $i$ и $j$.

Рассмотрим среду внутри цилиндра, состоящую из анизотропных осцилляторов, характеризуемых набором собственных частот $\omega_{L}, \omega_{r}$ и $\omega_{s}$. Такой среде соответствует кристалл, тензор диэлектрической проницаемости которого имеет диагональный вид с компонентами $\varepsilon_{\perp}$ и $\varepsilon_{\|}$, где индексы „, $\perp$ “ и , ||“ указывают на свойства материала в перпендикулярном и параллельном направлениях к оптической оси кристалла соответственно. Будем полагать, что частотные зависимости $\varepsilon_{\perp}(\omega)$ и $\varepsilon_{\|}(\omega)$ имеют вид [6-9]

$$
\varepsilon_{\perp}(\omega)=\varepsilon_{0}-\frac{\omega_{L}^{2}}{\omega^{2}-\omega_{r}^{2}}, \quad \varepsilon_{\|}(\omega)=\varepsilon_{0}-\frac{\omega_{L}^{2}}{\omega^{2}-\omega_{s}^{2}},
$$

где $\varepsilon_{0}-$ фоновое значение диэлектрической проницаемости кристалла, определяемое как высокочастотный предел $\varepsilon_{\perp}(\omega)$ и $\varepsilon_{\|}(\omega)$. Очевидно, что существуют такие области частот, в которых $\varepsilon_{\perp}(\omega)$ и $\varepsilon_{\|}(\omega)$ имеют отрицательные значения.

Продольные (аксиальные) спектральные составляющие поля имеют вид $[2,10,11]$

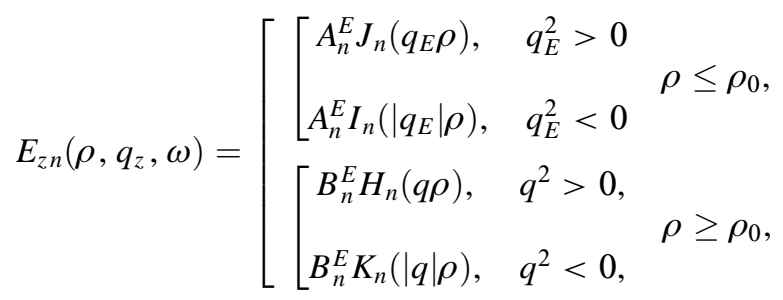

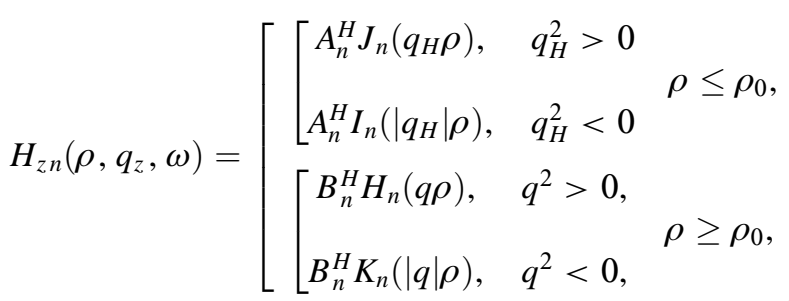

в которых $J_{n}(u)$ и $H_{n}(u)$ - цилиндрические функции $n$-го порядка Бесселя и Ганкеля, а $I_{n}(u)$ и $K_{n}(u)-$ модифицированные функции Бесселя $n$-го порядка первого (функция Инфельда) и второго (функция Макдональда) рода соответственно [12]; $A_{n}^{E, H}$ и $B_{n}^{E, H}-$ произвольные постоянные; $q_{H}=\sqrt{\varepsilon_{\perp} \omega^{2} / c^{2}-q_{z}^{2}}$ и $q_{E}=q_{H} \sqrt{\varepsilon_{\|} / \varepsilon_{\perp}}-$ поперечные (радиальные) волновые числа $H$ - и $E$-типов волн в цилиндре соответственно; $c$ - скорость света в вакууме; $q=\sqrt{\omega^{2} / c^{2}-q_{z}^{2}}-$ поперечное волновое число в вакууме. Радиальные зависимости составляющих $E_{z n}\left(\rho, q_{z}, \omega\right)$ и $H_{z n}\left(\rho, q_{z}, \omega\right)$ обусловлены конечностью поля при $\rho \rightarrow 0$ и $\rho \rightarrow \infty$. На частотах $\omega>0$ функция $H_{n}(q \rho) \equiv H_{n}^{(1)}(q \rho)$, а на частотах $\omega<0$ $H_{n}(q \rho) \equiv H_{n}^{(2)}(q \rho)$. Постоянные $B_{n}^{E}$ и $B_{n}^{H}$ выражаются соответственно через $A_{n}^{E}$ и $A_{n}^{H}$ из условий непрерывностей $E_{z n}\left(\rho, q_{z}, \omega\right)$ и $H_{z n}\left(\rho, q_{z}, \omega\right)$ составляющих на поверхности цилиндра $\rho=\rho_{0}$.

Поперечные компоненты электромагнитного поля собственных волн цилиндрического твердотельного волновода определяются через продольные $E_{z n}\left(\rho, q_{z}, \omega\right)$ и $H_{z n}\left(\rho, q_{z}, \omega\right)$, выражения для которых приведены, например, в работах $[10,11,13]$.

Удовлетворяя граничным условиям на поверхности цилиндра $\rho=\rho_{0}$, получим следующее дисперсионное уравнение для собственных волн твердотельного цилиндра $[3,14,15]$ :

$$
a_{n}^{2}-\Delta_{n}^{H} \Delta_{n}^{E}=0
$$

где

$$
\begin{gathered}
\Delta_{n}^{H}=\frac{1}{q_{H} \rho_{0}} \frac{J_{n}^{\prime}\left(q_{H} \rho_{0}\right)}{J_{n}\left(q_{H} \rho_{0}\right)}-\frac{1}{q \rho_{0}} \frac{H_{n}^{\prime}\left(q \rho_{0}\right)}{H_{n}\left(q \rho_{0}\right)}, \\
\Delta_{n}^{E}=\frac{\varepsilon_{\|}}{q_{E} \rho_{0}} \frac{J_{n}^{\prime}\left(q_{E} \rho_{0}\right)}{J_{n}\left(q_{E} \rho_{0}\right)}-\frac{1}{q \rho_{0}} \frac{H_{n}^{\prime}\left(q \rho_{0}\right)}{H_{n}\left(q \rho_{0}\right)}, \\
a_{n}=\frac{\omega q_{z} n}{c q_{H}^{2} q^{2} \rho_{0}^{2}}\left(\varepsilon_{\perp}-1\right) .
\end{gathered}
$$

Штрих у цилиндрических функций обозначает их производные по аргументу. Решения уравнения (2) определяют собственные частоты $\omega_{n s}=\omega_{n s}^{\prime}-i \omega_{n s}^{\prime \prime}$, где $\omega_{n s}^{\prime \prime} \geq 0$, цилиндрического волновода с модами $H$ - и $E$-типов. Функция $H_{n}\left(q \rho_{0}\right)$ при $\omega_{n s}^{\prime}>0$ является функцией Ганкеля первого рода $H_{n}^{(1)}\left(q \rho_{0}\right)$, а при $\omega_{n s}^{\prime}<0-$ функцией Ганкеля второго рода $H_{n}^{(2)}\left(q \rho_{0}\right)$. Азимутальный модовый индекс $n=0,1,2,3, \ldots$ соответствует половине количества вариаций поля по углу $\varphi$. Радиальный индекс $s$ отображает количество вариаций поля вдоль радиальной координаты $\rho$. В случае симметричных азимутально-однородных $(n=0)$ волн и аксиальнооднородных $\left(q_{z}=0\right)$ колебаний модовые индексы $s$ соответствуют порядковым номерам корней однородных дисперсионных уравнений, на которые распадается уравнение (2).

Дисперсионные зависимости $\omega_{0 s}\left(q_{z}\right)$ собственных симметричных $H_{0 s^{-}}$и $E_{0 s}$-волн твердотельного цилиндра определяются решениями дисперсионных уравнений $\Delta_{0}^{H}=0$ и $\Delta_{0}^{E}=0$ соответственно. Решения уравнения (2) при $n \neq 0$ определяют дисперсионные зависимости $\omega_{n s}\left(q_{z}\right)$ собственных гибридных $E H$ ( $H$-типа) или $H E$ ( $E$-типа)-волн волновода. Однозначное соответствие решений уравнения (2) определенному типу волны $(H$ или $E)$ можно идентифицировать только после определения доминирующей продольной компоненты поля, т.е. сравнения максимальных значений модулей $\left|H_{z n}\left(\rho, q_{z}, \omega_{n s}\right)\right|$ и $\left|E_{z n}\left(\rho, q_{z}, \omega_{n s}\right)\right|$. В случае $H E$-волны постоянная $A_{n}^{H}$ определяется через $A_{n}^{E}$, а в случае $E H$-волны - наоборот: $A_{n}^{E}$ через $A_{n}^{H}$.

Мы сосредоточим внимание только на собственных волнах твердотельного цилиндра, которые в вакууме имеют поверхностный характер. Для этих волн 
Виды собственных волн твердотельного цилиндра, расположенного в вакууме

\begin{tabular}{|c|c|c|c|c|c|}
\hline \multicolumn{2}{|c|}{$\begin{array}{c}\text { Знак квадрата } \\
\text { поперечного числа }\end{array}$} & \multicolumn{2}{|c|}{$\begin{array}{c}\text { Знак диэлектрической } \\
\text { проницаемости }\end{array}$} & \multirow[t]{2}{*}{ Виды волн } & \multirow[t]{2}{*}{ Ссылка } \\
\hline$q_{H}^{2}$ & $q_{E}^{2}$ & $\varepsilon_{\perp}(\omega)$ & $\varepsilon_{\|}(\omega)$ & & \\
\hline+ & + & + & + & объемно-поверхностные & {$[16]$} \\
\hline+ & - & + & - & $\begin{array}{c}\text { поверхностные и/или объемно-поверхностные } \\
\text { симметричные; } \\
\text { псевдоповерхностные гибридные }\end{array}$ & {$[16-18]$} \\
\hline- & + & + & - & объемно-поверхностные & {$[16]$} \\
\hline- & + & - & + & объемно-поверхностные & {$[16]$} \\
\hline- & - & - & - & поверхностные & {$[17,18]$} \\
\hline- & - & + & + & не существуют (запрещенная зона) & \\
\hline
\end{tabular}

выполняется условие $q^{2}<0$, что равносильно $q_{z}^{2}>\left(\omega_{n s} / c\right)^{2}$. Именно такие волны возбуждаются потоком заряженных частиц при выполнении условия резонанса Вавилова-Черенкова [3]. В этом случае в уравнении (2) слагаемые $H_{n}^{\prime}\left(q \rho_{0}\right) / q \rho_{0} H_{n}\left(q \rho_{0}\right)$ в $\Delta_{n}^{H}$ и $\Delta_{n}^{E}$ приобретают вид $-K_{n}^{\prime}\left(|q| \rho_{0}\right) /|q| \rho_{0} K_{n}\left(|q| \rho_{0}\right)$, а радиальные распределения продольных спектральных составляющих поля в вакууме описываются функциями Макдональда. В случае собственных волн цилиндра с поперечными волновыми числами, для которых выполняются условия $q_{H}^{2}<0$ и $q_{E}^{2}<0$, слагаемые $J_{n}^{\prime}\left(q_{H} \rho_{0}\right) / q_{H} \rho_{0} J_{n}\left(q_{H} \rho_{0}\right) \quad$ и $\quad \varepsilon_{\|} J_{n}^{\prime}\left(q_{E} \rho_{0}\right) / q_{E} \rho_{0} J_{n}\left(q_{E} \rho_{0}\right)$ в $\Delta_{n}^{H}$ и $\Delta_{n}^{E}$ в уравнении (2) соответственно приобретают вид $-I_{n}^{\prime}\left(\left|q_{H}\right| \rho_{0}\right) /\left|q_{H}\right| \rho_{0} I_{n}\left(\left|q_{H}\right| \rho_{0}\right) \quad$ и $-\varepsilon_{\|} I_{n}^{\prime}\left(\left|q_{E}\right| \rho_{0}\right) /\left|q_{E}\right| \rho_{0} I_{n}\left(\left|q_{E}\right| \rho_{0}\right)$.

В зависимости от знаков $q_{H}^{2}$ и $q_{E}^{2}$ собственные волны волновода имеют разные виды (см. таблицу). В таблице классификация волн приведена в соответствии с терминологиями работ [16-18].

Отметим, что собственные псевдоповерхностные аксиально-однородные $\left(q_{z}=0\right)$ колебания и псевдоповерхностные симметричные азимутально-однородные $(n=0)$ волны не существуют, так как они являются гибридными. Несуществование собственных волн структуры определяется отсутствием решений дисперсионного уравнения (2). В этом случае соответствующие области частот и волновых чисел формируют запрещенные зоны в спектрах собственных волн структуры.

\section{Численный анализ дисперсионного уравнения}

Отметим, что дисперсионное уравнение (2) приведено в безразмерном виде, что подчеркивает его универсальность. При этом безразмерность собственных частот волновода обеспечивается их нормированием на характеристическую частоту $\omega_{0}=c / \rho_{0}$, учитывающую идентичность цилиндрической конфигурации волновода.
Нами был исследован цилиндрический твердотельный волновод $\mathrm{c}$ характеристической частотой $\omega_{0}=6 \cdot 10^{10} \mathrm{~s}^{-1}$, что соответствует радиусу $\rho_{0}=0.5 \mathrm{~cm}$. В качестве материала волновода были использованы искусственные среды с параметрами: $\varepsilon_{0}=2, \omega_{L} / \omega_{0}=3.5$, $\omega_{r} / \omega_{0}=4, \omega_{s}=0$ или $\omega_{s} / \omega_{0}=6$.

На рис. 1 приведены дисперсионные зависимости собственных симметричных $(n=0)$ и гибридных $(n \neq 0)$ мод твердотельного цилиндра из искусственного материала, в котором собственная частота осцилляторов в продольном направлении $\omega_{s}=0$, представляющие решения уравнения (2). Линии 1 соответствуют частотам и продольным волновым числам, при которых поперечные волновые числа $q_{H}$ обращаются в нуль. Прямая 2 представляет световую линию в вакууме $\omega / \omega_{0}=q_{z} \rho_{0}$, что соответствует $q=0$. Для нас представляет интерес область частот и продольных волновых чисел собственных волн волновода, в которой $q^{2}<0$. Прямые 3 и 4 отображают частоты $\omega_{\|}$и $\omega_{\perp}$, на которых $\varepsilon_{\|}(\omega)$ и $\varepsilon_{\perp}(\omega)$ равны нулю соответственно. Прямая 5 соответствует нормированной собственной частоте осцилляторов $\omega_{r} / \omega_{0}$ искусственного материала в перпендикулярном направлении к оси симметрии цилиндра. Линии 6 и 7 представляют спектры собственных объемно-поверхностных волн $E$-типа, у которых квадраты поперечных волновых чисел $q_{E}^{2}>0$ и $q_{H}^{2}<0$ : симметричных $E_{0} 2^{-}$и $E_{0} 3^{-}$(рис. $\left.1, a\right)$ и гибридных $H E_{1} 1^{-}$и $H E_{1} 2^{-}$(рис. $\left.1, b\right)$ волн соответственно. Отметим, что в диапазоне частот $\omega<\omega_{\|}$частоты собственных мод волновода $\omega_{n s}$ с ростом радиального индекса уменьшаются. При $\omega_{n s} \rightarrow+0$ (сверху) плотность дисперсионных ветвей увеличивается. Штриховые дисперсионные ветви 8 соответствуют волнам $E$-типа: поверхностным симметричным $E$-волнам, у которых $q_{E}^{2}<0$ (рис. $\left.1, a\right)$, и псевдоповерхностным гибридным $H E_{1}$ 1-волнам, у которых $q_{E}^{2}<0$ и $q_{H}^{2}>0$ (рис. $1, b$ ). При этом компоненты тензора диэлектрической проницаемости цилиндра $\varepsilon_{\|}(\omega)<0$ и $\varepsilon_{\perp}(\omega)>0$. Ветвь поверхностных $E$-волн непрерывно переходит в ветвь объемно-поверхностных $E_{0}$ 1-волн, у которых $q_{E}^{2}>0$, 

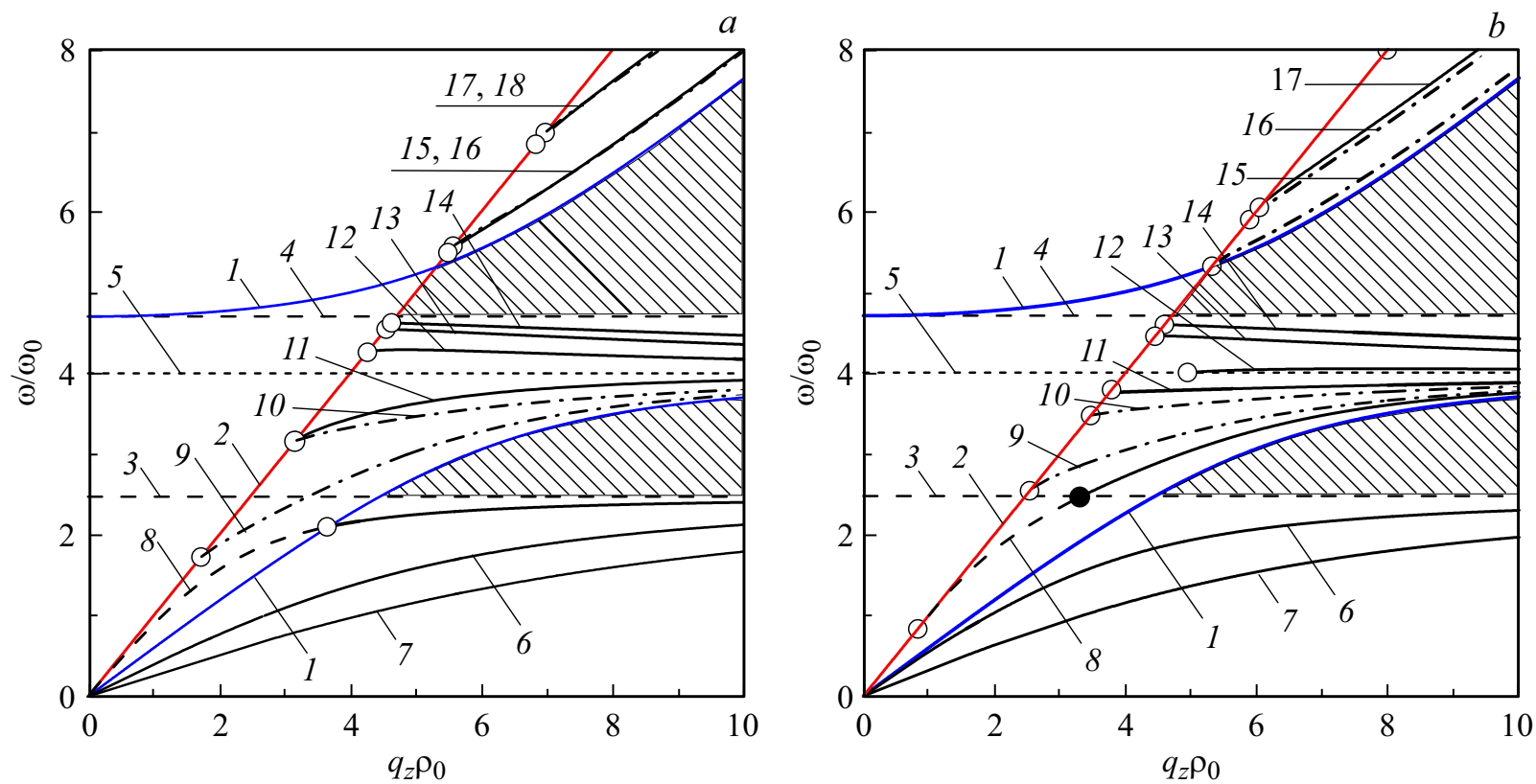

Рис. 1. Дисперсионные зависимости собственных симметричных $(a)$ и гибридных $(b)$ мод анизотропного твердотельного цилиндра, в котором частота собственных осцилляторов в продольном направлении $\omega_{s}=0$.

а ветвь псевдоповерхностных $H E_{11}$-волн - в ветвь объемно-поверхностных $H E_{1}$-волн, у которых $q_{E}^{2}>0$ и $q_{H}^{2}>0$, поскольку в соответствующей области частот $\varepsilon_{\|}(\omega)>0$. Штрихпунктирные линии 9 и 10 отображают дисперсионные зависимости объемно-поверхностных волн $H$-типа: симметричных $H_{0} 1$ и $H_{0} 2$ соответственно на рис. $1, a$ и гибридных $E H_{1} 1$ и $E H_{12}$ на рис. $1, b$. Сплошные линии 11 соответствуют объемноповерхностным волнам $E$-типа: симметричным $E_{0} 1$ на pис. $1, a$ и гибридным $H E_{1} 1$ модам на рис. $1, b$. Отметим, что в диапазоне частот $\omega_{\|}<\omega<\omega_{r}$ плотность дисперсионных зависимостей собственных мод $H$-типа с ростом радиального индекса $s$ увеличивается при $\omega_{n s} \rightarrow \omega_{r}-0$ (снизу). При этом волновое число $q_{H}$, входящее в аргумент функции Бесселя $J_{0}\left(q_{H} \rho\right)$ в дисперсионном уравнении $\Delta_{0}^{H}=0$, изменяется от конечного значения $q_{H}$ при $\omega=\omega_{\|}$до $q_{H} \rightarrow \infty$ при $\omega=\omega_{r}$. В этом же частотном диапазоне число собственных мод $E$-типа остается конечным. Это связано с тем, что поперечное волновое число $q_{E}$, входящее в аргумент функции Бесселя $J_{0}\left(q_{E} \rho_{0}\right)$ в дисперсионном уравнении $\Delta_{0}^{E}=0$, изменяется от $q_{E}=0$ при $\omega=\omega_{\|}$до $q_{E}=\omega_{r} \sqrt{\varepsilon_{\|}\left(\omega_{r}\right)} / c$ при $\omega=\omega_{r}$. Линии 12-14 представляют спектры объемноповерхностных симметричных $E_{0 s^{-}}$(рис. 1,a) и гибридных $H E_{1 s^{-}}$(рис. 1,b) мод с $s=1-3$ в диапазоне $\omega_{r}<\omega<\omega_{\perp}$. В этом частотном диапазоне плотность дисперсионных кривых волновода также увеличивается с ростом радиального индекса $s$ собственных мод при приближении их частот $\omega_{n s}$ к частоте $\omega_{\perp}$ снизу. Кривые 15-18 на рис. $1, a$ отображают дисперсионные зависимости объемно-поверхностных $E_{0} 1^{-}, H_{01^{-}}$, $E_{0} 2^{-}$и $H_{0}$ 2-волн соответственно, причем сплошные линии для волн $E$-типа, а штрихпунктирны - для $H$-типа. На рис. $1, b$ дисперсионные зависимости 15-17 соответствуют объемно-поверхностным $E H_{1} 1^{-}, E H_{1} 2^{-}$ и $H E_{1}$ 1-волнам соответственно в порядке роста частот $\omega_{n s}$. Пустыми кружками показаны точки начала (окончания) спектров соответствующих волн. Значения $\omega / \omega_{0}$ и $q_{z} \rho_{0}$ в этих точках, вообще говоря, не удовлетворяют дисперсионному уравнению (2).

Из рис. 1 видно, что в диапазоне частот $\omega_{r}<\omega<\omega_{\perp}$ собственные объемно-поверхностные волны $E$-типа твердотельного цилиндра (кривые $12-14$ ) обладают аномальной дисперсией, а их дисперсионные зависимости при $q_{z} \rho_{0} \rightarrow \infty$ асимптотически приближаются к прямой $\omega / \omega_{0}=\omega_{r} / \omega_{0}+0$ (сверху). Отметим, что в частотных диапазонах $\omega_{r}<\omega<\omega_{\perp}$ и $\omega<\omega_{\|}$в исследуемом волноводе не существуют собственные симметричные волны $H$-типа, поскольку уравнение $\Delta_{0}^{H}=0$ не имеет решений. Собственные параметры волновода с симметричными $E_{0 s}$-волнами определяются решениями дисперсионного уравнения $\Delta_{0}^{E}=0$. Вне частотного диапазона $\omega_{r}<\omega<\omega_{\perp}$ собственные волны волновода обладают нормальной дисперсией. В диапазоне $\omega_{\|}<\omega<\omega_{r}$ при $q_{z} \rho_{0} \rightarrow \infty$ дисперсионные кривые собственных объемно-поверхностных волн асимптотически приближаются к прямой $\omega / \omega_{0}=\omega_{r} / \omega_{0}-0$ (снизу), а в диапазоне $\omega<\omega_{\|}-$к прямой $\omega / \omega_{0}=\omega_{\|} / \omega_{0}-0$ (снизу). Отметим, что в диапазоне $\omega<\omega_{\|}$существуют поверхностные, псевдоповерхностные и объемноповерхностные волны одной частоты, отличающиеся волновыми числами. Подобное существование поверхностных и объемно-поверхностных волн одной частоты было обнаружено ранее в левосторонних средах $[19,20]$.

На рис. 2 приведены спектры собственных симметричных $(n=0)$ и гибридных $(n \neq 0)$ мод цилиндра из искусственного материала, в котором нормированная 

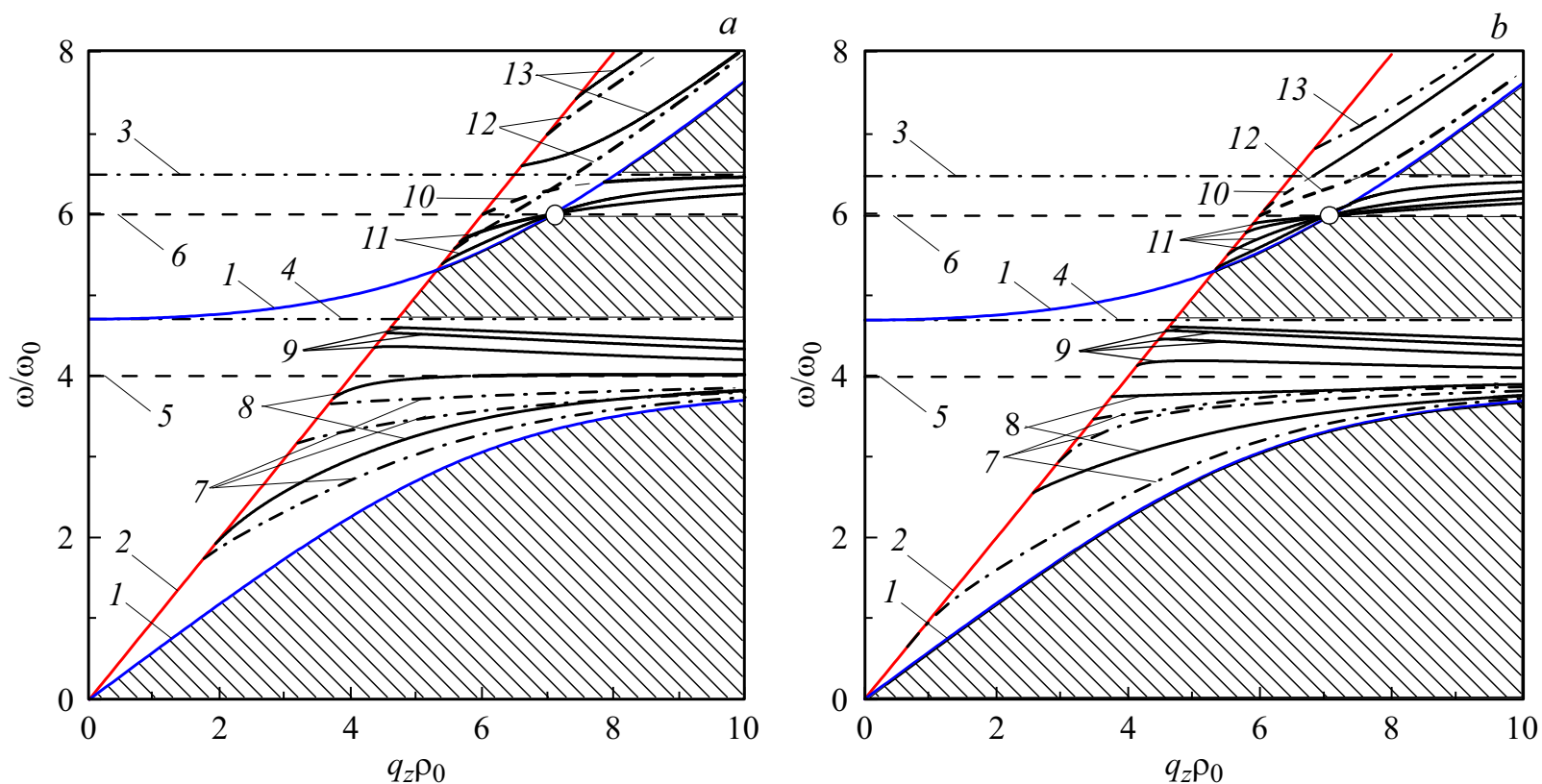

Рис. 2. Дисперсионные зависимости собственных симметричных $(a)$ и гибридных $(b)$ мод цилиндра из материала, в котором собственная частота осцилляторов $\omega_{s}=6 \omega_{0}$.

собственная частота осцилляторов в продольном направлении $\omega_{s} / \omega_{0}=6$. Линии $1-5$ идентичны линиям на рис. 1. Как и ранее, сосредоточим внимание на области частот и продольных волновых чисел собственных волн волновода, в которой $q^{2}<0$. Прямая 6 соответствует нормированной собственной частоте осцилляторов $\omega_{s} / \omega_{0}$ искусственного материала вдоль оси симметрии цилиндра. Линии 7 и 8 представляют спектры объемноповерхностных волн $H$ - и $E$-типов, у которых квадраты поперечных волновых чисел $q_{E}^{2}>0$ и $q_{H}^{2}>0$ : симметричных $H_{0} 1^{-}, E_{0} 1^{-}, H_{0} 2^{-}, H_{0} 3^{-}$и $E_{02^{-}}$(рис. $2, a$ ) и гибридных $E H_{11^{-}}, H E_{11^{-}}, E H_{12^{-}}, E H_{13^{-}}$и $H E_{12^{-}}$ (рис. $2, b$ ) волн, соответственно расположенных в порядке роста частот $\omega_{n s}$. Отметим, что в диапазоне частот $0<\omega<\omega_{r}$ плотность дисперсионных зависимостей собственных мод $H$-типа с ростом радиального индекса увеличивается при $\omega_{n s} \rightarrow \omega_{r}-0$ (снизу). При этом волновое число $q_{H}$, входящее в аргумент функции Бесселя $J_{0}\left(q_{H} \rho_{0}\right)$ в дисперсионном уравнении $\Delta_{0}^{H}=0$, изменяется от $q_{H}=0$ при $\omega=0$ и $q_{z}=0$ до $q_{H} \rightarrow \infty$ при $\omega=\omega_{r}$. В этом же частотном диапазоне число собственных мод $E$-типа остается конечным. Это связано с тем, что поперечное волновое число $q_{E}$, входящее в аргумент функции Бесселя $J_{0}\left(q_{E} \rho_{0}\right)$ в дисперсионном уравнении $\Delta_{0}^{E}=0$, изменяется от $q_{E}=0$ при $\omega=0$ и $q_{z}=0$ до $q_{E}=\omega_{r} \sqrt{\varepsilon_{\|}\left(\omega_{r}\right)} / c$ при $\omega=\omega_{r}$. Кривые 9 отображают дисперсионные зависимости объемноповерхностных симметричных $E_{0 s^{-}}$(рис. 2,a) и гибридных $H E_{1 s^{-}}$(рис. 2,b) мод с $s=3-6$ в диапазоне $\omega_{r}<\omega<\omega_{\perp}$. При этом плотность дисперсионных кривых собственных мод волновода увеличивается с ростом $s$ при приближении их частот $\omega_{n s}$ к частоте $\omega_{\perp}$ снизу. Штриховые участки дисперсионных ветвей 10 в частотном диапазоне $\omega_{s}<\omega<\omega_{\|}$соответствуют по- верхностным симметричным волнам $E$-типа на рис. 2, $a$ и псевдоповерхностным гибридным $H E_{1} 1$-волнам на рис. $2, b$, у которых $q_{E}^{2}<0$ и $q_{H}^{2}>0$. При этом компоненты тензора диэлектрической проницаемости цилиндра $\varepsilon_{\|}(\omega)<0$ и $\varepsilon_{\perp}(\omega)>0$. Ветвь поверхностных волн переходит с разрывом на зависимости $q_{H}=0$ в ветвь объемно-поверхностных $E_{0}$ 1-волн, у которых $q_{E}^{2}>0$. В свою очередь, ветвь псевдоповерхностных $H E_{1}$ 1-волн переходит непрерывно в ветвь объемно-поверхностных $H E_{1}$ 1-волн в диапазоне $\omega>\omega_{\|}$. Линии 11 представляют спектры объемно-поверхностных симметричных $E_{0 s^{-}}$(рис. 2,a) и гибридных $H E_{1 s^{-}}$(рис. 2, $b$ ) мод. В диапазоне $\omega_{\perp}<\omega<\omega_{s} E_{0} 1^{-}$и $H E_{1}$ 1-моды имеют наименьшие значения частот, а в диапазоне $\omega_{s}<\omega<\omega_{\|}$ $E_{02^{-}}$и $H E_{1} 1_{\text {-моды }}$ - наибольшие. При этом в диапазоне $\omega_{\perp}<\omega<\omega_{s}$ плотность дисперсионных кривых собственных мод волновода увеличивается с ростом $s$ при $\omega_{n s} \rightarrow \omega_{s}-0$, когда $\varepsilon_{\|}(\omega) \rightarrow+\infty$, а в диапазоне $\omega_{s}<\omega<\omega_{\|}$при $\omega_{n s} \rightarrow \omega_{s}+0$, когда $\varepsilon_{\|}(\omega) \rightarrow-\infty$. Переход дисперсионных ветвей из одного частотного диапазона в другой осуществляется через нулевую запрещенную зону (точка пересечения ветвей и зависимости $q_{H}=0$ на рис. 2) [21]. При переходе ветвей симметричных мод осуществляется трансформация радиальных индексов с увеличением на единицу в направлении роста частот $\omega_{0 s}$. Семейства дисперсионных кривых 12 на рис. 2, a отображают спектры собственных объемноповерхностных симметричных $H_{0 s}$-волн с $s=1,2$ в порядке роста частот $\omega_{0 s}$. Дисперсионная ветвь 12 на рис. $2, b$ в частотном диапазоне $\omega_{s}<\omega<\omega_{\|}$соответствует псевдоповерхностным (штриховая линия), а в диапазоне $\omega>\omega_{\|}$- объемно-поверхностным (штрихпунктирная линия) гибридным $E H_{1}$ 1-волнам. Непрерывный переход ветви из одного частотного диапазона 
в другой осуществляется с трансформацией псевдоповерхностных волн в объемно-поверхностные воны на частоте $\omega_{\|}$, на которой $\varepsilon_{\|}=0$. Линии 13 представляют дисперсионные зависимости объемно-поверхностных симметричных $E_{0 s}$-волн $(s=1,2$ в порядке роста частот $\left.\omega_{0 s}\right)$ на рис. $2, a$ и гибридных $E H_{1}$ 2-волн на рис. $2, b$.

Из рис. 2 видно, что в диапазоне частот $\omega_{r}<\omega<\omega_{\perp}$ объемно-поверхностные волны $E$-типа (кривые 9) обладают отрицательными групповыми скоростями, а их дисперсионные зависимости при $q_{z} \rho_{0} \rightarrow \infty$ асимптотически приближаются к прямой $\omega / \omega_{0}=\omega_{r} / \omega_{0}+0$. В этом частотном диапазоне в цилиндре не существуют собственные $H_{0 s}$-волны, поскольку уравнение $\Delta_{0}^{H}=0$ не имеет решений. Частоты и продольные волновые числа симметричных $E_{0 s}$-волн определяются решениями дисперсионного уравнения $\Delta_{0}^{E}=0$. Вне диапазона $\omega_{r}<\omega<\omega_{\perp}$ собственные волны волновода обладают положительными групповыми скоростями. В диапазоне $\omega<\omega_{r}$ при $q_{z} \rho_{0} \rightarrow \infty$ дисперсионные кривые собственных объемно-поверхностных волн асимптотически приближаются к прямой $\omega / \omega_{0}=\omega_{r} / \omega_{0}-0$, а в диапазоне $\omega_{s}<\omega<\omega_{\|}-$к прямой $\omega / \omega_{0}=\omega_{\|} / \omega_{0}-0$. Отметим, что в диапазоне $\omega_{s}<\omega<\omega_{\|}$существуют поверхностные, псевдоповерхностные и объемно-поверхностные волны одной частоты, отличающиеся волновыми числами.

Заштрихованные зоны на рис. 1 и 2 отображают области частот и волновых чисел, с которыми в исследуемых волноводах собственные волны не существуют (запрещенные зоны). В этих зонах соответствующие дисперсионные уравнения (2) не имеют решений.

Качественное поведение дисперсионных кривых анизотропного цилиндра с собственными модами, имеющими азимутальные индексы $n>1$, аналогично дисперсионным зависимостям мод с $n=1$.

Отметим, что на практике, когда материал цилиндра обладает потерями, в волноводе, как правило, проявляются собственные моды с радиальными индексами $s=1,2$, а остальные затухают [10,22]. В действительности слабое затухание собственных мод волновода обеспечивается сосредоточением их полей вблизи цилиндрической поверхности волновода. Такими свойствами обладают моды с азимутальными индексами $n \gg 1$, например, „шепчущей галереи“ в квазиоптических структурах $[10]$

Собственные объемно-поверхностные волны $E$-типа исследуемых цилиндрических волноводов в диапазонах $\omega_{r}<\omega<\omega_{\perp}$ обладают отрицательными групповыми скоростями, что способствует возникновению абсолютной неустойчивости при их возбуждении цилиндрическим трубчатым электронным пучком [2]. Эти волны существуют в анизотропном волноводе с диэлектрическими проницаемостями $\varepsilon_{\|}>0$ и $\varepsilon_{\perp}<0$, что обеспечивает $q_{E}^{2}>0$ и $q_{H}^{2}<0$.

\section{Заключение}

В работе теоретически изучены свойства собственных волн анизотропного цилиндрического твердотельного волновода, изготовленного из материала, у которого компоненты тензора диэлектрической проницаемости обладают частотной дисперсией. Приведена классификация собственных волн волновода, расположенного в вакууме. Определены спектры собственных симметричных и гибридных мод цилиндров из анизотропных искусственных материалов. Обнаружены объемноповерхностные волны $E$-типа, которые в некотором частотном диапазоне обладают отрицательными групповыми скоростями. Показано, что существование таких волн обусловлено анизотропными свойствами волновода, у которого диэлектрические проницаемости $\varepsilon_{\perp}<0$ и $\varepsilon_{\|}>0$, что однозначно обеспечивает $q_{H}^{2}<0$ и $q_{E}^{2}>0$. При возбуждении волн с отрицательными групповыми скоростями электронным потоком в системе возможно возникновение абсолютной неустойчивости [2]. Показано существование в цилиндрическом твердотельном волноводе собственных поверхностных симметричных волн $E$-типа и псевдоповерхностных гибридных волн $E$ - и $H$-типов. Установлены области частот, в которых одновременно существуют поверхностные, псевдоповерхностные и объемно-поверхностные волны одной частоты, отличающиеся волновыми числами. Отмечены области частот и волновых чисел, в которых в исследуемых волноводах собственные волны не существуют (запрещенные зоны).

\section{Список литературы}

[1] Дормидонтов А.В., Кириченко А.Я., Лонин Ю.Ф., Пономарев А.Г., Прокопенко Ю.В., Сотников Г.В., Уваров В.Т., Филиппов Ю.Ф. // Письма в ЖТФ. 2012. Т. 38. Вып. 2. C. $65-73$.

[2] Аверков Ю.О., Прокопенко Ю.В., Яковенко В.М. // Радиофизика и электроника. 2016. Т. 7 (21). Вып. 2. С. 28-35.

[3] Аверков Ю.О., Прокопенко Ю.В., Яковенко В.М. // Радиофизика и электроника. 2016. Т. 7 (21). Вып. 4. С. 68-76.

[4] Силин Р.A., Рухадзе А.A. Электромагнитные свойства плазмы и плазмоподобных сред. М.: Госатомиздат, 1961. $244 \mathrm{c}$.

[5] Александров А.Ф., Богданкевич Л.С., Рухадзе А.А. Основы электродинамики плазмы. М.: Высшая школа, 1978. 407 с.

[6] Пабомов В.E. // Труды Физического ин-та им. Лебедева. 1961. T. 16. C. $94-139$.

[7] Силин Р.А. // УФН. 2006. Т. 175. № 5. С. 562-565.

[8] Дмитрук Н.Л., Литовченко В.Г., Стрижевский В.Л. Поверхностные поляритоны в полупроводниках и диэлектриках. Киев: Наук. думка, 1989. 376 с.

[9] Коломенский А. // Доклады АН СССР. 1952. Т. 86. № 6. C. 1097-1099.

[10] Кириченко А.Я., Прокопенко Ю.В., Филиппов Ю.Ф., Черпак Н.Т. Квазиоптические твердотельные резонаторы. Киев: Наук. думка, 2008. 286 с.

[11] Akay M.F., Prokopenko Yu., Kharkovsky S. // Microw. Optic. Technol. Lett. 2004. Vol. 40. N 2. P. 96-101.

[12] Справочник по специальным функциям. С формулами, графиками и таблицами / Под. ред. М. Абрамовица, И. Стиган. М.: Наука, 1979. 832 с. 
[13] Ильченко М.Е., Взятышев В.Ф., Гассанов Л.Г. идр. Диэлектрические резонаторы / Под ред. М.Е. Ильченко. М.: Радио и связь, 1989. 328 с.

[14] Прокопенко Ю.В., Филиппов Ю.Ф. // ЖТФ. 2002. Т. 72. Вып. 6. С. 79-84.

[15] Дормидонтов А.В., Прокопенко Ю.В. // Изв. вузов. Радиофизика. 2013. Т. 56. Вып. 6. С. 428-442.

[16] Кузелев М.В., Рухадзе А.А., Стрелков П.С. Плазменная релятивистская СВЧ-электроника. М.: Изд-во МГТУ им. Н.Э. Баумана, 2002. 544 с.

[17] Бразис Р.С. // Литовский физический сборник. 1981. Т. 21. № 4. C. 73-117.

[18] Белецкий Н.Н., Светличный В.М., Халамейда Д.Д., Яковенко В.М. Электромагнитные явления СВЧ в неоднородных полупроводниковых структурах. Киев: Наук. думка, 1991. $215 \mathrm{c}$.

[19] Ruppin R. // J. Phys.: Conden. Matter. 2001. Vol. 13. N 9. P. 1811-1819.

[20] Averkov Yu.O., Yakovenko V.M. // Phys. Rev. B. 2005. Vol. 72. N 20. P. 205110.

[21] Виноградов А.П., Дорофеенко А.В., Мерзликин А.М., Лисянский А.А. // УФН. 2010. Т. 180. Вып. 3. С. 249-263.

[22] Barannik A., Cherpak N., Kirichenko A., Prokopenko Yu., Vitusevich S., Yakovenko V. // Intern. J. Microw. Wireless Technol. 2017. Vol. 9. N 4. P. 781-796. 